DOI: 10.20472/IAC.2018.044.032

\author{
RAHUL MUKHERJEE \\ Indian Institute of Management Calcutta, India
}

\title{
CAUSAL INFERENCE FROM STRIP-PLOT DESIGNS: METHODOLOGY AND APPLICATIONS IN A POTENTIAL OUTCOMES FRAMEWORK
}

\begin{abstract}
:
Randomization based causal inference in a potential outcomes framework has been of significant interest in recent years. The principal advantage of such inference is that it does not involve rigid model assumptions. We develop the methodology of causal inference from strip-plot designs that are very useful when the responses are influenced by treatments having a factorial structure and the factor levels are hard-to-change, so that they have to be applied to larger clusters of experimental units. Our results can have application to diverse fields such as sociology, agriculture, and urban management, to name only a few.
\end{abstract}

For example, in an agricultural field experiment with two factors, irrigation and harvesting, both requiring larger plots, the experimental units can be laid out in several blocks, each block being a rectangular array of rows and columns. One can then employ a strip-plot design that randomizes the methods of irrigation among the rows and the methods of harvesting among the columns, in each block. Similarly, a strip-plot design is a natural choice in urban traffic management where each block is rectangular grid of streets, and within any such grid, signaling conditions are randomized among the north-south streets while traffic rules are randomized among east-west streets.

With a strip-plot design under a potential outcomes framework, we propose an unbiased estimator for any treatment contrast and work out an expression for its sampling variance. We next obtain a conservative estimator of the sampling variance. This conservative estimator has a nonnegative bias, and becomes unbiased under a condition that is much milder than the age-old Neymannian strict additivity. A minimaxity property of this variance estimator is also established. Simulation results on the coverage of resulting confidence intervals lend support to theoretical considerations.

\section{Keywords:}

Between-block additivity; conservative variance estimator; minimaxity; treatment contrast; unbiased estimator.

JEL Classification: C10, C14 